\title{
FINITE UNIONS OF CONVEX SETS ${ }^{1}$
}

\author{
J. F. LAWRENCE, W. R. HARE, JR. AND JOHN W. KENELLY ${ }^{2}$
}

ABSTRACT. In this paper it is shown that a set is the union of $k$ convex subsets if and only if every finite subset of it is contained in some $k$ convex subsets of it. This is a characterization of a set as the union of a finite number of convex sets by conditions on its finite subsets.

Also, a proof of McKinney's theorem for unions of two convex sets is given using similar methods.

Richard McKinney has given a characterization of unions of two convex sets. In this paper a complete characterization of unions of convex sets is given, and we give another proof of McKinney's result.

Here, a $k$-partition of a set $S$ is a family $\mathscr{S}=\left\{S_{1}, S_{2}, \cdots, S_{k}\right\}$ of subsets of $S$, having $k$ elements, where $S_{i} \cap S_{j}=\varnothing$ for $i \neq j$, and $\bigcup_{i=1}^{k} S_{i}=S$.

A property $P$ of sets is said to be hereditary if, given any set $S$ with property $P$, any subset $T \subset S$ has property $P$.

I. First, we prove a theorem which enables us to by-pass further reference to Zorn's Lemma.

THEOREM 1. Let $P$ be a hereditary property of sets. Let $S$ be a set such that for every finite subset $F \subset S$, there is a $k$-partition of $F,\left\{F_{1}, F_{2}, \cdots, F_{k}\right\}$, such that $F_{i}$ has property $P, 1 \leqq i \leqq k$. Then there is a $k$-partition of $S$, $\left\{S_{1}, S_{2}, \cdots, S_{k}\right\}$, such that for every finite subset $F \subset S, F \cap S_{i}$ has property $P, 1 \leqq i \leqq k$.

Proof. Let $\mathscr{F}=\{\alpha \subset S \mid \alpha$ is finite $\}$; and, for each $\alpha \in \mathscr{F}$, let $P_{\alpha}=$ $\left\{\left(F_{1}, \cdots, F_{k}\right) \mid F_{i}\right.$ has property $P, 1 \leqq i \leqq k$, and $\left\{F_{1}, \cdots, F_{k}\right\}$ is a $k$ partition of $\alpha\}$. Since $\alpha$ is finite, $P_{\alpha}$ is finite and, with the discrete topology, $P_{\alpha}$ is a compact topological space. The Tychonoff theorem gives $\prod_{\alpha \in \mathscr{F}} P_{\alpha}$ to be compact. (For $X \in \prod_{\alpha \in \mathscr{F}} P_{\alpha}$, denote the $\alpha$ th coordinate of $X$ by $X_{\alpha}$.

Received by the editors August 18, 1970.

AMS 1969 subject classifications. Primary 5230.

Key words and phrases. Unions of convex sets, convex kernel.

1 This research was supported in part by a National Science Foundation grant GY-7473 (Undergraduate Research Participation). The first author was a student participant from Oklahoma State University and the remaining authors were supervisors of the project.

${ }^{2}$ The authors wish to express appreciation to the referee for the method of proving Theorem 1; it significantly shortened and clarified the proof. 
Also, $X_{\alpha}$ is a $k$-tuple of subsets of $S$ and the $i$ th is denoted $X_{\alpha i}, 1 \leqq i \leqq k$.) For $\alpha \in \mathscr{F}$ define

$$
A_{\alpha}=\left\{X \in \prod_{\alpha \in \mathscr{F}} P_{\alpha} \mid \text { if } \beta \subset \alpha, \text { then } X_{\beta i}=X_{\alpha i} \cap \beta, 1 \leqq i \leqq k\right\} .
$$

It is easy to verify that $A_{\alpha}$ is closed for each $\alpha \in \mathscr{F}$ and it is now shown that, likewise, each $A_{\alpha} \neq \varnothing$. Since $P_{\beta} \neq \varnothing$, by hypothesis, select a $Q_{\beta} \in P_{\beta}$ for each $\beta \in \mathscr{F}$. Then, for $\alpha \in \mathscr{F}$ let

$$
\begin{aligned}
R_{\beta i}^{\alpha} & =Q_{\beta i} & & \text { if } \beta \notin \alpha, \\
& =Q_{\alpha i} \cap \alpha & & \text { if } \beta \subset \alpha ;
\end{aligned}
$$

then it is immediate that the point $R^{\alpha}$ thus defined is in $A_{\alpha}$. Next, observe that

$$
\varnothing \neq A_{\alpha_{1} \cup \cdots \cup \alpha_{n}} \subset A_{\alpha_{1}} \cap \cdots \cap A_{\alpha_{n}}
$$

for any positive integer $n$. Thus, the family $\left\{A_{\alpha}\right\}_{\alpha \in \mathscr{F}}$ is a collection of nonempty, closed subsets of the compact space $\prod_{\alpha \in \mathscr{F}} P_{\alpha}$ having the finite intersection property. It follows that there exists an $X \in \bigcap_{\alpha \in \mathscr{F}} A_{\alpha}$.

Now define $S_{i}=\left\{x \in S \mid\{x\}=X_{\{x\} i}\right\}$. It follows easily that for each $\alpha \in \mathscr{F}, S_{i} \cap \alpha=X_{\alpha i}$. It is also routine to see that $\left\{S_{1}, \cdots, S_{k}\right\}$ partitions $S$. Since $S_{i} \cap \alpha=X_{\alpha i}$, it follows that $S_{i} \cap \alpha$ has property $P$, so $\left\{S_{1}, \cdots, S_{k}\right\}$ is the desired partition of $S$.

II. THEOREM 2. Let $S$ be a subset of a linear space such that each finite subset $F \subset S$ has a k-partition, $\left\{F_{1}, F_{2}, \cdots, F_{k}\right\}$, where conv $F_{i} \subset S$, $1 \leqq i \leqq k$. Then $S$ is the union of $k$ convex sets.

Proof. Let a set $T \subset S$ have property $P$ if and only if conv $T \subset S$. This property is hereditary, and any finite subset $F \subset S$ has a $k$-partition, $\left\{F_{1}, F_{2}, \cdots, F_{k}\right\}$, such that each $F_{i}$ has property $P$. By Theorem $1, S$ has a $k$-partition, $\left\{S_{1}, S_{2}, \cdots, S_{k}\right\}$, such that for any finite subset $F \subset S$, $F \cap S_{i}$ has property $P, 1 \leqq i \leqq k$. In particular, if $F$ is a finite subset of $S_{i}$, conv $F \subset S$. This implies that conv $S_{i} \subset S$, since

$$
\text { conv } S_{i}=\bigcup\left\{\operatorname{conv} F \mid F \text { is a finite subset of } S_{i}\right\} \text {. }
$$

We have

$$
S=\bigcup_{i=1}^{k} S_{i}=\bigcup_{i=1}^{k}\left(\operatorname{conv} S_{i}\right)
$$

We prove the following statement of McKinney's theorem:

TheOREM 3. Let $S$ be a closed subset of a topological linear space such that for every finite subset $F \subset S$, there is a 2-partition $\left\{F_{1}, F_{2}\right\}$ such that if $x, y \in F_{i}(1 \leqq i \leqq 2)$ then $x y \subset S$. Then $S$ is the union of two convex sets. 
Proof. Let the set $T \subset S$ have property $P$ if and only if, for any $x$, $y \in T, x y \subset S$. This property is hereditary, and any finite subset $F \subset S$ has a 2-partition $\left\{F_{1}, F_{2}\right\}$ such that $F_{1}$ and $F_{2}$ have property $P$. By Theorem 1 , $S$ has a 2-partition, $\left\{S_{1}, S_{2}\right\}$, such that for any finite subset $F \subset S, F \cap S_{1}$ and $F \cap S_{2}$ have property $P$. Clearly, $S_{1} \cup S_{2}=S$; for $x, y \in S_{1}, x y \subset S$; and for $x, y \in S_{2}, x y \subset S$.

For $x \in S$, let st $x=\{y \in S \mid x y \subset S\}$. Let $\left\{y_{i}\right\}_{i=1}^{\infty}$ be a sequence in st $x$ with limit $y_{0} ; y_{0} \in S$ since $S$ is closed. For any element $\lambda x+(1-\lambda) y_{0}$ $(0 \leqq \lambda \leqq 1)$ of $x y_{0}$, the sequence $\left\{\lambda x+(1-\lambda) y_{i}\right\}_{i=1}^{\infty}$ is in $S$ and has limit $\lambda x+(1-\lambda) y_{0}$, so $\lambda x+(1-\lambda) y_{0}$ is in $S$. Therefore $x y_{0} \subset S, y_{0} \in$ st $x$, and st $x$ is closed.

Let $A=\bigcap_{x \in S_{1}}$ (st $x$ ) and $B=\bigcap_{x \in S_{2}}$ (st $\left.x\right) ; S_{1} \subset A$ and $S_{2} \subset B$, so $A \cup B=$ $S_{1} \cup S_{2}=S$. We want to show that $A$ and $B$ are convex.

Clearly, $A$ and $B$ are closed sets, and $A \cap B$ is the convex kernel of $S$.

Let $a_{1}$ and $a_{2}$ be elements of $A$. Either both $a_{1}$ and $a_{2}$ are in $S_{2}$, or at least one is in $S_{1}$ and the other is in $A=\bigcap_{x \in S_{1}}($ st $x)$. In either case, $a_{1} a_{2} \subset S$. It is necessary to show that $a_{1} a_{2} \subset A$.

Let $c$ be an element of $a_{1} a_{2}$ other than $a_{1}$ and $a_{2}$. If $c \notin A$ then $c \in B$. Since $A$ is closed, $c$ is in an open-ended segment of $a_{1} a_{2}$, say $\left(b_{1} b_{2}\right)$, such that $\left(b_{1} b_{2}\right) \subset S-A$ and $b_{1}, b_{2} \in A$. But $\left(b_{1} b_{2}\right) \subset S-A \subset B$, and since $B$ is closed $b_{1}, b_{2} \in B$. We have $b_{1}, b_{2} \in A \cap B$, the convex kernel of $S$. But this means $c \in A \cap B \subset A$.

Therefore, $a_{1} a_{2} \subset A$ for $a_{1}, a_{2} \in A$, so $A$ is convex. Similarly, $B$ is convex, and $S=A \cup B$.

III. Theorem 1 also gives an immediate proof of the next theorem. The easy details are omitted.

THEOREM 4. Let $G=(V, E)$ be a graph, possibly infinite, with no multiple edges or loops. Then $G$ has chromatic number $k$ if and only if every finite induced subgraph of $G$ has chromatic number at most $k$, and there is at least one such subgraph whose chromatic number is precisely $k$.

Various efforts (see [1], [2], [3], [4]) have been made to characterize finite unions of convex sets by simple conditions on finite subsets of points in the resulting set. The McKinney theorem is the first such characterization for $k=2$. In all these prior efforts, however, the basic appoach has been through conditions about segments (convex hulls of pairs of points) rather than convex hulls of larger finite sets. The present approach eventually presents itself as a very "natural" method and yields a reasonable characterization.

\section{REFERENCES}

1. W. R. Hare, Jr. and John W. Kenelly, Sets expressible as unions of two convex sets, Proc. Amer. Math. Soc. 25 (1970), 379-380. MR 41 \#2528. 
2. Richard L. McKinney, On unions of more than two convex sets, Notices Amer. Math. Soc. 17 (1970), 249. Abstract \#672-575.

3. - On unions of two convex sets, Canad. J. Math. 18 (1966), 883-886. MR 34 \#1923.

4. F. A. Valentine, A three point convexity property, Pacific J. Math. 7 (1957), 1227-1235. MR 20 \#6071.

Department of Mathematical Sciences, Clemson University, Clemson, South Carolina 29631 